\title{
Endoscopic Trans-Turbinal Medial Maxillectomy: A Modified Endoscopic Medial Maxillectomy Technique to Preserve the Inferior Turbinate
}

\author{
Ki Ju Cho ${ }^{1}$, Hyun-Jin Cho ${ }^{1,2}$, Yeon-Hee Joo ${ }^{3}$, Yung Jin Jeon ${ }^{1,2}$, \\ Sea-Yuong Jeon ${ }^{4}$, and Sang-Wook Kim ${ }^{1,2}$ (D) \\ ${ }^{1}$ Department of Otorhinolaryngology, Gyeongsang National University Hospital \& Gyeongsang National University College of Medicine, \\ Jinju; and ${ }^{2}$ Institute of Health Sciences, Gyeongsang National University, Jinju, and; \\ ${ }^{3}$ Department of Otorhinolaryngology, Gyeongsang National University Changwon Hospital, Changwon, and; \\ ${ }^{4}$ Yeil ENT Clinic, Changwon, Korea
}

내시경적 경비갑개 내상악절제술: 하비갑개를 보존하는 내시경적 내상악절제술의 새로운 방법

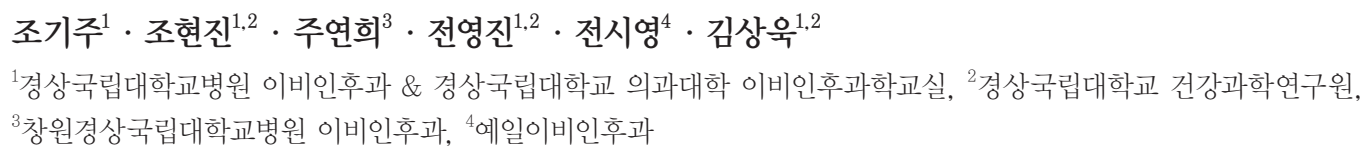

\author{
Received July 13, 2021 \\ Revised October 29, 2021 \\ Accepted November 1, 2021 \\ Address for correspondence \\ Sang-Wook Kim, MD, PhD \\ Department of Otorhinolaryngology, \\ Gyeongsang National University \\ Hospital, 79 Gangnam-ro, \\ Jinju 52727, Korea \\ Tel $+82-55-750-8177$ \\ Fax $+82-55-759-0613$ \\ E-mail basilent@gnu.ac.kr
}

Endoscopic medial maxillectomy (EMM) and its modifications are surgical techniques are used to treat recalcitrant maxillary sinusitis as well as maxillary sinus tumors. In this report, we propose a simple and efficient modification of EMM, called endoscopic trans-turbinal medial maxillectomy (ETTMM), by which the inferior turbinate (IT), nasolacrimal duct, and anatomical integrity of the nasal valve area are preserved. A total of 10 patients (five tumorous and five nontumorous maxillary diseases) underwent ETTMM. Briefly, a turbinate mucosal flap on the superior aspect of the IT was elevated after middle meatal antrostomy. Then a trans-turbinal window was developed to expose the inferior meatus, after which an extended maxillary antrostomy was generated. Finally, the turbinate mucosal flap was repositioned after complete removal of the antral lesions. All lesions were successfully treated using ETTMM. Our modification was easy to perform, and we achieved good endoscopic visualization and accessibility throughout the whole antrum by creating a trans-turbinal window and extended maxillary antrostomy. We could perform postoperative surveillance easily through the wide antrostomy using rigid endoscopes of various angles. ETTMM is a simple and useful modification of EMM that provides clear visualization and great accessibility to most aspects of the maxillary antrum while preserving the nasal functional units, including the IT and nasal valve area. Korean J Otorhinolaryngol-Head Neck Surg 2021;64(12):959-64

Keywords Maxillary sinus; Natural orifice endoscopic surgery; Organ preservation; Surgical procedure, Turbinates.

\section{Introduction}

Endonasal endoscopic procedures have replaced many open nasal surgeries since their introduction in the late 1980s for the treatment of various sinonasal tumors as well as inflammatory sinonasal diseases. ${ }^{1,2)}$ External approaches, including the canine fossa puncture or Caldwell-Luc approaches, however, are commonly combined with the endonasal approach

This is an Open Access article distributed under the terms of the Creative Commons Attribution Non-Commercial License (https://creativecommons.org/licenses/by-nc/4.0) which permits unrestricted non-commercial use, distribution, and reproduction in any medium, provided the original work is properly cited. 
to treat maxillary sinus lesions such as antrochoanal polyps, inverted papilloma, and severely diseased maxillary sinus. ${ }^{3,4)}$ This is because surgeons treating maxillary sinus lesions, particularly inverted papilloma or recalcitrant sinusitis, require direct visualization, sufficient working space for manipulation, and possible access to all aspects of the antrum.

Endoscopic maxillary mega-antrostomy and endoscopic medial maxillectomy (EMM) are endonasal surgical techniques for treating refractory maxillary sinusitis and maxillary sinus tumors, respectively, without an external approach. ${ }^{5,6)}$ EMM can be used for lesions involving the zygomatic recess, anterior wall, or floor of the antrum and includes removal of the inferior turbinate (IT), medial maxillary wall, and nasal valve area. ${ }^{6}$ Preservation of the IT and anatomical integrity of the nasal valve area should be considered because these units are important in nasal physiology in terms of humidification and dynamics of nasal air flow.

To preserve the IT in situ and not violate the nasal valve area when performing EMM, we developed a novel technique called endoscopic trans-turbinal medial maxillectomy (ETTMM). Using this technique, we could access most aspects of the antrum from the middle and inferior meatuses through the extended antrostomy under direct visual control while preserving the IT in situ. In addition to achieving good intraoperative visualization and accessibility, we attained satisfactory postoperative results in 10 cases of various tumorous and nontumorous maxillary lesions. Here we present the procedures and results.

\section{Methods}

ETTMM was performed by two experienced surgeons (S.W.K. and S.Y.J.) in 10 patients from October 2014 to October 2017. We conducted a retrospective chart review of these patients. This study was approved by the Institutional Review Board of Gyeongsang National University Hospital (IRB 2017-12-008).

\section{Surgical technique (Supplementary Video 1)}

Uncinectomy is followed by a middle meatal antrostomy (MMA). In cases of revision, residual uncinate process and any scar in the area of the maxillary sinus ostium are removed (Fig. 1A and B). MMA is extended inferiorly to the IT and can be extended posteriorly to the posterior wall of the maxillary sinus and superiorly to the orbital floor depending on the extent of the maxillary lesions. Then a short vertical extension incision is made to the IT at the junction of the frontal process of the maxilla and the IT along the maxillary line (Fig. 1B). A posteroinferior-based triangular mucosal flap is developed on the posterior two thirds of the IT (Fig. 1C). The exposed turbinate bone and underlying lateral-side mucosa of the IT are removed to create a trans-turbinal window to the upper inferior meatus (Fig. 1D). The IT is displaced medially to access the lower inferior meatus, and MMA is extended inferiorly to the nasal floor, posteriorly to the posterior wall of the antrum, and anteriorly to the pyriform aperture to make a sufficiently large surgical corridor to control the antral lesions. The created large antrostomy lie posterior to the nasolacrimal duct and the Hasner's valve (Fig. 1E). Hasner's valve should be preserved under endoscopic visualization. In cases where the Hasner's valve is not easily found, the bulb press test is conducted to identify tears oozing out from the nasolacrimal system. If needed, a fine ball probe is used to confirm the location of the Hasner's valve. The antrum is accessed through the middle meatus and trans-turbinal window above the IT and through the inferior meatus below the IT. After the antral lesions are controlled and thoroughly irrigated, the inferior and middle meatuses are secured with absorbable nasal packs. Finally, the triangular mucosal flap is repositioned towards the remaining IT and secured with a strip of oxidized regenerated cellulose (Surgicel; Johnson \& Johnson Medical Korea Ltd., Seoul, Korea) (Fig. 1F).

\section{Results}

The mean age of the patients was 61.0 years (range, 48-87 years, with a male:female ratio of 8:2). Five patients received ETTMM to treat nontumorous lesions in the maxillary sinus, such as recalcitrant maxillary sinusitis, dental implant-related maxillary sinusitis, postoperative cheek cyst (POCC) in the zygomatic recess (Fig. 2A and B), and inflammatory antral polyps (Fig. 2C and D). The other five patients underwent ETT$\mathrm{MM}$ to resect tumors arising from the antrum, including cavernous hemangioma, inverted papilloma (Fig. 2E and F), and locally recurrent myxofibrosarcoma (Fig. 2G and H). All lesions were successfully addressed using ETTMM. The mean follow-up duration was 17.4 months (range, 3-35 months). We performed postoperative disease surveillance using rigid endoscopes of various angles (Fig. 3A-D) and/or imaging studies such as $\mathrm{CT}$ (Fig. 3E and F). Recirculation of a mucus was observed in no cases. Disease recurrence was observed in a single patient who had undergone ETTMM for recurrent myxo- 

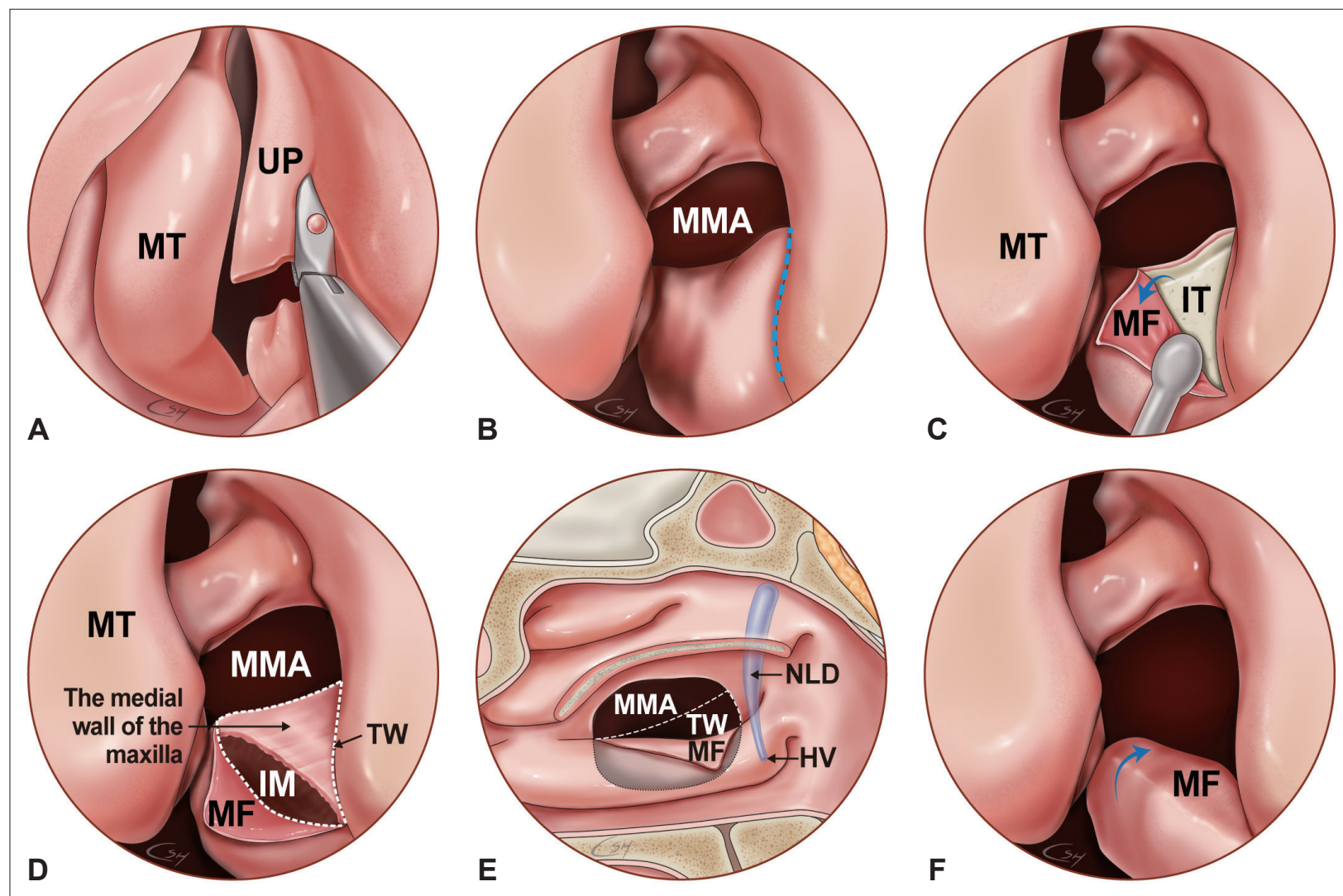

Fig. 1. Schematic illustration of endoscopic trans-turbinal medial maxillectomy. After uncinectomy $(A)$ and MMA, vertical mucosal incision (black dotted line) is made on the IT mucosa along the anterior margin of the MMA (B). After mucosal flap elevation, the bony portion of the IT is exposed (C). The turbinate bones are removed to create a trans-turbinal window (TW; blue dotted line), and the medial wall of the maxilla and the upper inferior meatus are exposed (D). The MMA is extended inferiorly to create large maxillary antrostomy (translucent gray area), which lies posteriorly to nasolacrimal duct and Hasner's valve (E; the MT is expressed as if it is partially resected to describe the large antrostomy from the medial point of view). After the antral lesions are controlled, the triangular mucosal flap on the IT is repositioned and secured with a strip of oxidized regenerated cellulose $(F)$. The maxillary antrum can be endoscopically visualized via a large entry site $(M M A+T W)$ in the middle meatus $(E$ and $F)$. MT, middle turbinate; UP, uncinate process; MMA, middle meatal antrostomy; MF, mucosal flap; IT, inferior turbinate; IM, inferior meatus; TW, trans-turbinal window; NLD, nasolacrimal duct; HV, Hasner's valve.
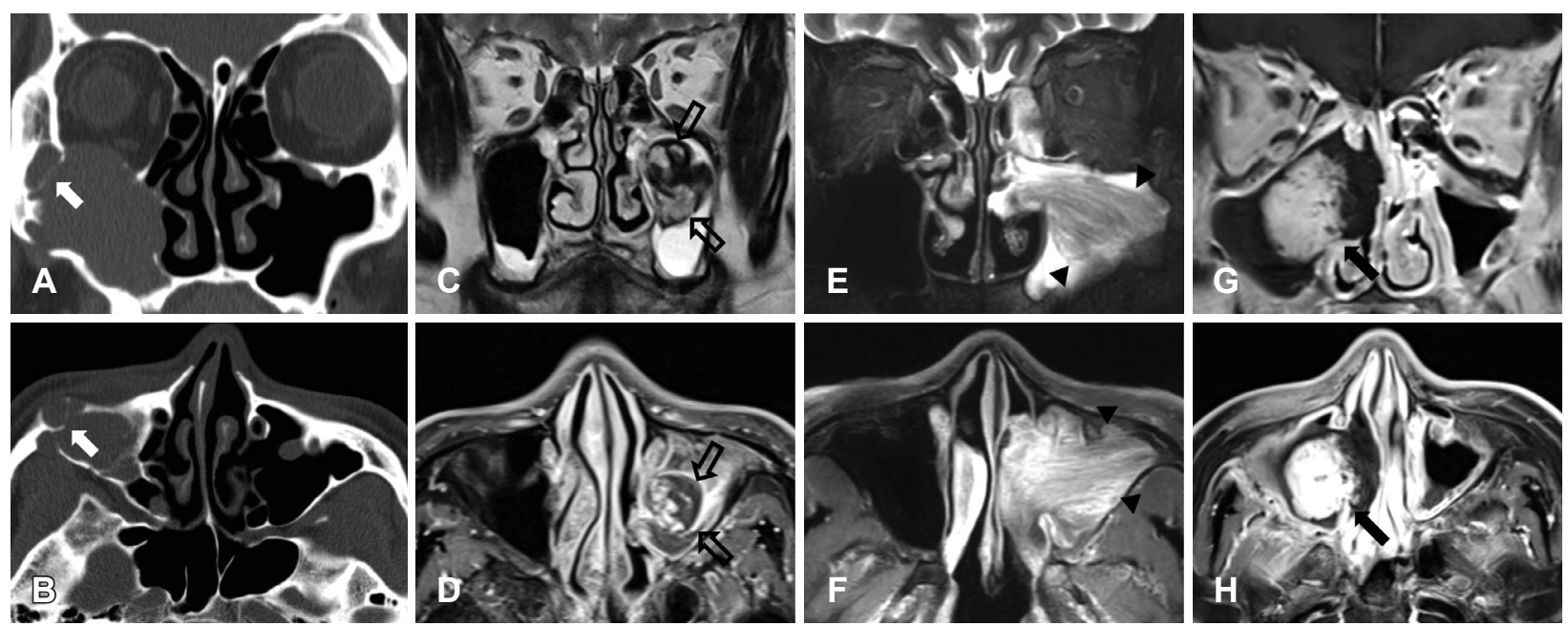

Fig. 2. Representative cases who received endoscopic trans-turbinal medial maxillectomy. Computerized tomography scans of a postoperative cheek cyst (white arrow) in the zygomatic recess (A and B). T2-weighted (C) and gadolinium-enhanced T1-weighted (D) MR images of inflammatory antral polyps (clear arrows). T2-weighted (E) and gadolinium-enhanced T1-weighted (F) MR images of inverted papilloma arising from the zygomatic recess (black arrowheads). Gadolinium-enhanced T1-weighted magnetic resonance images of recurrent myxofibrosarcoma (black arrow) arising from the posteromedial wall of the maxillary sinus with a small stalk $(G$ and $H)$. 
fibrosarcoma 14 months postoperatively (Table 1).

\section{Discussion}

Since its introduction, EMM has become a preferred endonasal surgical approach in many cases of inverted papilloma spreading throughout the maxillary sinus. When it was first introduced, the IT and NLD were resected depending on the area involved and the pathology of the lesion. ${ }^{6}$ Because the IT performs important functions in nasal physiology, including warming and humidifying inhaled air, some modifications to preserve the IT have been introduced. ${ }^{7,8)}$ Some modifications involved cutting the head of the IT, positioning the IT medially or posteriorly during surgery, and repositioning the IT at the original site. ${ }^{7,8)}$ In other modifications, called the medial shift of IT or prelacrimal approaches, a vertical incision was made more anteriorly on the mucosa of the lateral nasal wall along the anterior margin of the IT. These modifications included os-
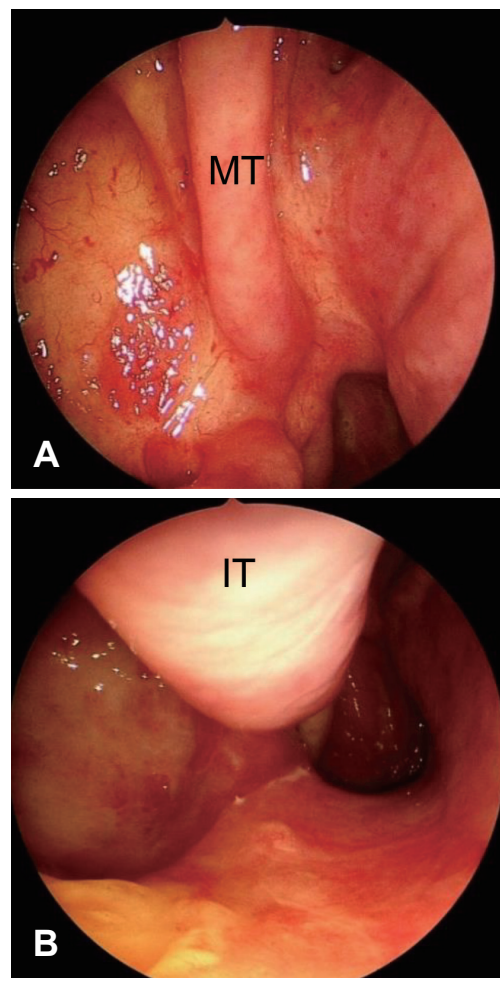
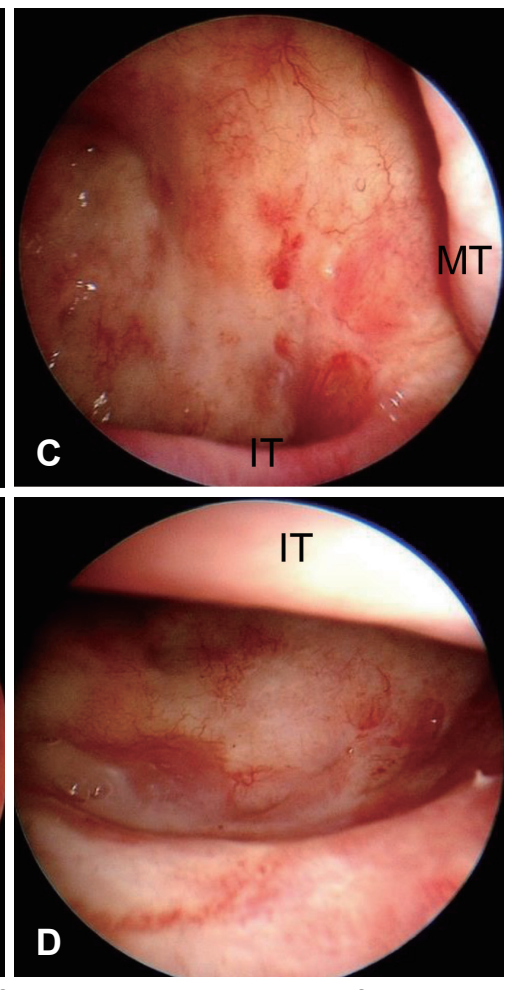

Fig. 3. Representative demonstration of postoperative surveillance after endoscopic trans-turbinal medial maxillectomy. Nasal photographs obtained by $0^{\circ}(\mathrm{A}$ and $\mathrm{B})$ and $70^{\circ}(\mathrm{C}$ and $\mathrm{D})$ rigid endoscopes in a case of recurrent myxofibrosarcoma. Six months after surgery, the clear maxillary antrum was well visualized through the middle meatus ( $A$ and $C$ ) and inferior meatus (B and $D)$. Postoperative computerized tomography scans of a patient with inverted papilloma arising from the zygomatic recess ( $E$ and $F$ ). The IT in the middle of the maxillary antrostomy is well visualized (arrow). MT, middle turbinate; IT, inferior turbinate.

Table 1. Characteristics of the subjects

\begin{tabular}{cccllll}
\hline Case & Age & Sex & \multicolumn{1}{c}{ Diagnosis } & Side & Follow-up duration (month) & Follow-up results \\
\hline 1 & 70 & M & Inflammatory antral polyps & Left & 26 & No recurrence \\
2 & 40 & F & CRS, recurred & Both & 13 & Controlled \\
3 & 65 & M & Dental implant-related sinusitis & Left & 27 & Controlled \\
4 & 48 & M & POCC & Right & 11 & Controlled \\
5 & 51 & M & POCC & Left & 3 & Controlled \\
6 & 62 & M & Inverted papilloma & Right & 17 & No recurrence \\
7 & 67 & $M$ & Inverted papilloma & Left & 16 & No recurrence \\
8 & 68 & $M$ & Inverted papilloma & Left & 8 & No recurrence \\
9 & 60 & F & Cavernous hemangioma & Right & 35 & No recurrence \\
10 & 60 & $M$ & Recurred myxofibrosarcoma & Right & 18 & Recurred at postoperative 14 month \\
\hline
\end{tabular}

CRS, chronic rhinosinusitis; POCC, postoperative cheek cyst 
teotomy of the medial maxillary wall, identification, and preservation of the NLD. ${ }^{9-12)}$ Using these modifications, the prelacrimal recess of the maxillary antrum was opened and access to the maxillary antrum was achieved through the anterior side of the preserved NLD. Despite the changes in a more functional direction, however, these modifications violate the anatomical integrity of the nasal valve area through mucosal incisions and removal of bone at the anterior end of the IT, which may result in nasal functional disturbance.

Extended maxillary antrostomy or maxillary mega-antrostomy, which involves resecting the posterior half or greater portion of the IT, has been used to treat refractory maxillary sinus inflammatory lesions since its introduction in the late 1990s. ${ }^{5,13,14)}$ Because the scope of maxillary antrostomy has increased, including partial resection of the IT, its surgical procedures overlap with those of EMM. ${ }^{15)}$ Accordingly, EMM, including maxillary mega-antrostomy, is now considered a suitable surgical technique for treating recalcitrant maxillary sinusitis as well as for extirpating sinonasal tumors. ${ }^{16)}$

Our modified technique, ETTMM, involves elevating a turbinate mucosal flap on the superior aspect of the IT after MMA, developing a trans-turbinal window to expose the inferior meatus, extending MMA inferiorly to make an extended maxillary antrostomy, and then repositioning the turbinate mucosal flap after completely removing the antral lesions. The repositioned mucosal flap covers the remaining IT bone, and thus postoperative stenosis of the trans-turbinal window is minimized. Unlike the aforementioned IT-preserving techniques that rely on vertical mucosal incision at the head of the IT or lateral nasal wall, our technique does not disrupt the anatomical integrity of the nasal valve area. Moreover, our modification is easy to perform and allows for a smooth transition from MMA to extended antrostomy because the posteroinferior margin of MMA and the inferior meatus are well visualized by the creation of the trans-turbinal window. The transturbinal window also provides more working space for surgical instruments such as microdebriders of various angled tips all over the antrum to the zygomatic recess compared to simple combination of MMA and inferior meatal antrostomy (IMA). While preserving the IT in situ, our technique achieves extended endoscopic visualization and unrestricted surgical manipulation of the zygomatic recess laterally, the antral floor inferiorly, and the anterior maxillary wall by two separate channels above and below the IT. Finally, the trans-turbinal window enables wider endoscopic visualization during follow-up than combination of MMA and IMA. Preventing the stenosis of the trans-turbinal window by mucosal flap coverage is a key procedure for this purpose.

One limitation of this technique is that tumors growing from the anterior region to the NLD, such as the prelacrimal recess and pyriform aperture, cannot be optimally addressed; thus, other techniques, such as the prelacrimal approach or CaldwellLuc approach, would be more suitable. However, given that removal of the frontal process of the maxilla included in some types of the prelacrimal approach ${ }^{17)}$ may potentially lead to nasal valve problems or facial asymmetry, our technique may be more appropriate in cases of maxillary tumors that do not involve the prelacrimal recess and pyriform aperture.

In our case series, a case of POCC in the zygomatic recess (Fig. 2A and B) and a case of inverted papilloma originating from the zygomatic recess (Figs. 2E, 2F, 3E, and 3F) showed good surgical accessibility of ETTMM to the zygomatic recess laterally. Although the lesions in these two cases were located far lateral in the maxillary sinus, the wide endoscopic view through the trans-turbinal window allowed clear postoperative surveillance. In other two cases (Fig. 2C and D, G and H), the origins of lesions were medial maxillary wall. The transturbinal window created during ETTMM provided simultaneous visualization of the maxillary sinus and the inferior meatus, and thus the masses could be resected in a safe and efficient manner. In the single case of recurrence after ETTMM, the first site of the tumor recurrence was the medial wall of the maxillary sinus (Fig. 2G and H). Because the tumors were pedunculated with a small stalk, they were successfully removed by ETTMM, including partial resection of the posterior region of the IT with clear resection margins. During the follow-up period, the maxillary antrum was well visualized using rigid endoscopes of various angles (Fig. 3A-D) until the second recurrence, which occurred at the posterior maxillary wall. Given the negative resection margins during ETTMM, the recurrence site (the posterior maxillary wall), and the 14-month interval between ETTMM and the second recurrence, the cause of the second recurrence was not ascribed to ETTMM.

In conclusion, in our experience, ETTMM appears to be a safe and efficient modification of EMM that provides clear visualization and great accessibility to all aspects of the maxillary antrum while preserving the IT in situ and the anatomical integrity of the nasal valve area.

\section{Supplementary Video Legend}

Video 1. Video of endoscopic trans-turbinal medial maxillectomy in a patient with refractory maxillary sinusitis who had twice undergone endoscopic sinus surgery. First revised MMA, which includes 
the removal of any scarring in the area of the natural ostium, is performed (a). Next a vertical incision is made on the IT at the junction of the frontal process of the maxilla and the IT, and a posteroinferior-based triangular mucosal flap is developed (b). The exposed turbinate bone and underlying lateral-side mucosa of the IT are removed to create a trans-turbinal window to the inferior meatus (c). In the inferior meatus, after the trans-turbinal window and the posteroinferior portion of MMA lying posterior to Hasner's valve are identified, extended antrostomy is performed (d). After the antral lesions are controlled, the triangular mucosal flap on the IT is repositioned and secured with a strip of oxidized regenerated cellulose (e). MMA, middle meatal antrostomy; IT, inferior turbinate.

\section{Supplementary Materials}

The Data Supplement is available with this article at https://doi. org/10.3342/kjorl-hns.2021.00654.

\section{Acknowledgments}

This work was supported by Biomedical Research Institute Fund (GNUHBRIF-2017-0007) from the Gyeongsang National University Hospital.

The illustration was supported by Suhyun Chae from National Cancer Center Korea.

\section{Author Contribution}

Conceptualization: Sea-Yuong Jeon. Data curation: all authors. Funding acquisition: Sang-Wook Kim. Supervision: Sea-Yuong Jeon, Sang-Wook Kim. Writing — original draft: Ki Ju Cho, Sang-Wook Kim. Writing — review \& editing: Ki Ju Cho, Sang-Wook Kim.

\section{ORCID}

Sang-Wook Kim https://orcid.org/0000-0002-1681-0556

\section{REFERENCES}

1) Stammberger H, Kopp W. Functional endoscopic sinus surgery: The Messerklinger technique. Philadelphia: B.C. Decker;1991.

2) Lund V, Howard DJ, Wei WI. Endoscopic resection of malignant tumors of the nose and sinuses. Am J Rhinol 2007;21(1):89-94.

3) Sathananthar S, Nagaonkar S, Paleri V, Le T, Robinson S, Wormald PJ. Canine fossa puncture and clearance of the maxillary sinus for the severely diseased maxillary sinus. Laryngoscope 2005;115(6): 1026-9.
4) Hong SK, Min YG, Kim CN, Byun SW. Endoscopic removal of the antral portion of antrochoanal polyp by powered instrumentation. Laryngoscope 2001;111(10):1774-8.

5) Cho DY, Hwang PH. Results of endoscopic maxillary megaantrostomy in recalcitrant maxillary sinusitis. Am J Rhinol 2008; 22(6):658-62.

6) Wormald PJ, Ooi E, van Hasselt CA, Nair S. Endoscopic removal of sinonasal inverted papilloma including endoscopic medial maxillectomy. Laryngoscope 2003;113(5):867-73.

7) Weber RK, Werner JA, Hildenbrand T. Endonasal endoscopic medial maxillectomy with preservation of the inferior turbinate. Am J Rhinol Allergy 2010;24(6):132-5.

8) Gras-Cabrerizo JR, Massegur-Solench H, Pujol-Olmo A, MontserratGili JR, Ademá-Alcover JM, Zarraonandia-Andraca I. Endoscopic medial maxillectomy with preservation of inferior turbinate: How do we do it? Eur Arch Otorhinolaryngol 2011;268(3):389-92.

9) Suzuki M, Nakamura Y, Nakayama M, Inagaki A, Murakami S, Takemura K, et al. Modified transnasal endoscopic medial maxillectomy with medial shift of preserved inferior turbinate and nasolacrimal duct. Laryngoscope 2011;121(11):2399-401.

10) Nakayama T, Asaka D, Okushi T, Yoshikawa M, Moriyama H, Otori N. Endoscopic medial maxillectomy with preservation of inferior turbinate and nasolacrimal duct. Am J Rhinol Allergy 2012;26(5): 405-8.

11) Zhou B, Han DM, Cui SJ, Huang Q, Wang CS. Intranasal endoscopic prelacrimal recess approach to maxillary sinus. Chin Med J (Engl) 2013;126(7):1276-80.

12) Morrissey DK, Wormald PJ, Psaltis AJ. Prelacrimal approach to the maxillary sinus. Int Forum Allergy Rhinol 2016;6(2):214-8.

13) Rodriguez MJ, Sargi Z, Casiano RR. Extended maxillary sinusotomy in isolated refractory maxillary sinus disease. Otolaryngol Head Neck Surg 2007;137(3):508-10.

14) Coleman JR Jr, Duncavage JA. Extended middle meatal antrostomy: The treatment of circular flow. Laryngoscope 1996;106(10):1214-7.

15) Wang EW, Gullung JL, Schlosser RJ. Modified endoscopic medial maxillectomy for recalcitrant chronic maxillary sinusitis. Int Forum Allergy Rhinol 2011;1(6):493-7.

16) Konstantinidis I, Constantinidis J. Medial maxillectomy in recalcitrant sinusitis: When, why and how? Curr Opin Otolaryngol Head Neck Surg 2014;22(1):68-74.

17) Suzuki M, Nakamura Y, Yokota M, Ozaki S, Murakami S. Modified transnasal endoscopic medial maxillectomy through prelacrimal duct approach. Laryngoscope 2017;127(10):2205-9. 